\title{
Purification of chemically fixed HIV-1 spikes for oriented display on nanoparticles
}

\author{
MB Zwick*, D Leaman \\ From AIDS Vaccine 2012 \\ Boston, MA, USA. 9-12 September 2012
}

\section{Background}

HIV-1 neutralizing antibodies (Abs) bind to the envelope glycoprotein (Env) spike, which functions as a trimer of gp120-gp41 heterodimers that is anchored in the viral membrane. However, Env trimers are low in copy number and coexist with irrelevant forms of Env and its byproducts, which typically elicit non-neutralizing Abs.

\section{Methods}

Here, we have attempted to generate immunogens by fixing trimeric spikes of HIV-1JR-FL using a defined chemical crosslinker, purifying the crosslinked spikes from virions, and immunodepleting them of irrelevant Env contaminants using non-neutralizing Abs.

\section{Results}

Purified, crosslinked spikes were bound by a panel of neutralizing Abs, but not by non-neutralizing Abs, and are virtually devoid of non-trimeric Env. However, at least one neutralizing epitope on gp120, the crown of V3, appears to be occluded by crosslinking. An immunization was performed using the purified, crosslinked Env trimers as a boosting agent following a DNA prime using full-length env, either as soluble protein or captured onto small proteoliposome nanoparticles (PLNs). The binding titers of the Ab response to crosslinked Env spikes were quite weak, possibly reflecting an overall weaker immune response. Boosting animals with trimer-PLNs however elicited a qualitatively different neutralizing $\mathrm{Ab}$ response than uncrosslinked Env on virions, with sporadic activity against neutralization-resistant HIV-1 isolate JR-CSF, reduced neutralization of sensitive Tier 1 isolates, and reduced antibody responses against host protein.

The Scripps Research Institute, La Jolla, CA, USA

\section{Conclusion}

Immunizing animals using purified, crosslinked Env spikes captured on PLNs elicited a qualitatively different and broader neutralizing $\mathrm{Ab}$ response than uncrosslinked, heterogeneous Env. We conclude that with further changes to the crosslinking and immunization strategy crosslinked and purified Env spikes hold promise as a vaccine candidate.

Published: 13 September 2012

doi:10.1186/1742-4690-9-S2-P347

Cite this article as: Zwick and Leaman: Purification of chemically fixed HIV-1 spikes for oriented display on nanoparticles. Retrovirology 20129 (Suppl 2):P347. and take full advantage of:

- Convenient online submission

- Thorough peer review

- No space constraints or color figure charges

- Immediate publication on acceptance

- Inclusion in PubMed, CAS, Scopus and Google Scholar

- Research which is freely available for redistribution 